\title{
Enhanced thermoelectric properties of Bi2Te3- based micro-nano fibers via thermal drawing and interfacial engineering
}

\section{Min Sun}

South China University of Technology https://orcid.org/0000-0002-7494-3584

\section{Guowu Tang}

South China University of Technology

\section{Hanfu Wang}

National Center for Nanoscience and Technology

\section{Ting Zhang}

Nanyang Technological University https://orcid.org/0000-0001-5967-0525

\section{Pengyu Zhang}

South China University of Technology

\section{Bin Han}

South China University of Technology

\section{Ming Yang}

Institute of Engineering Thermophysics, Chinese Academy of Science, Beijing 100190, P. R. China

\section{Hang Zhang}

Chinese Academy of Sciences

\section{Yicong Chen}

Sun Yat-sen University

\section{Jun Chen}

School of Electronics and Information Technology, Sun Yat-sen University https://orcid.org/0000-00017397-2714

\section{Dongdan Chen}

South China University of Technology

\section{Jiulin Gan}

State Key Laboratory of Luminescent Materials and Devices, Institute of Optical Communication Materials, South China University of Technology https://orcid.org/0000-0001-9842-0510

\section{Qi Qian}

South China University of Technology

\section{Zhongmin Yang ( $\nabla$ yangzm@scut.edu.cn )}

State Key Laboratory of Luminescent Materials and Devices, South China University of Technology, Guangzhou 510640, China 
Article

Keywords: thermoelectric fibers, Bi2Te3, thermal drawing, interfacial engineering

Posted Date: February 4th, 2022

DOI: https://doi.org/10.21203/rs.3.rs-1309115/v1

License: (c) (i) This work is licensed under a Creative Commons Attribution 4.0 International License. Read Full License 


\title{
Enhanced thermoelectric properties of $\mathrm{Bi}_{2} \mathrm{Te}_{3}$-based micro-nano fibers via thermal drawing and interfacial engineering
}

\author{
Min Sun ${ }^{1,2, \#, \text { Guowu Tang }}{ }^{1,2,3, \#}$, Hanfu Wang,"\#, Ting Zhang ${ }^{5,6}$, Pengyu Zhang ${ }^{2}$, Bin \\ Han $^{2}$, Ming Yang 5 , Hang Zhang ${ }^{5}$, Yicong Chen ${ }^{7}$, Jun Chen ${ }^{7}$, Dongdan Chen², Jiulin \\ $\mathrm{Gan}^{2}$, Qi Qian ${ }^{2, *}$, and Zhongmin Yang ${ }^{1,2, *}$ \\ ${ }^{1}$ School of Physics and Optoelectronics, South China University of Technology, Guangzhou 510640, China \\ ${ }^{2}$ State Key Laboratory of Luminescent Materials and Devices, Institute of Optical Communication Materials, Guangdong \\ Provincial Key Laboratory of Fiber Laser Materials and Applied Techniques, and Guangdong Engineering Technology Research \\ and Development Center of Special Optical Fiber Materials and Devices, School of Materials Science and Engineering, South \\ China University of Technology, Guangzhou 510640, China. \\ ${ }^{3}$ School of Physics and Optoelectronic Engineering, Guangdong University of Technology, Guangzhou 510006, China. \\ ${ }^{4}$ CAS Key Laboratory of Nanosystem and Hierarchical Fabrication, National Center for Nanoscience and Technology of \\ China, Beijing 100190, China. \\ ${ }^{5}$ Institute of Engineering Thermophysics, Innovation Academy for Light-duty Gas Turbine, Chinese Academy of Sciences, \\ Beijing 100190, China. \\ ${ }^{6}$ Nanjing Institute of Future Energy System, Nanjing 211135, China \\ ${ }^{7}$ State Key Laboratory of Optoelectronic Materials and Technologies, Guangdong Province Key Laboratory of Display Material \\ and Technology, School of Electronics and Information Technology, Sun Yat-sen University, Guangzhou, 510275 China. \\ \# The authors contributed equally: Min Sun, Guowu Tang, Hanfu Wang. \\ *E-mail:qianqi@scut.edu.cn; yangzm@scut.edu.cn.
}

\begin{abstract}
:
High-performance thermoelectric (TE) materials with great flexibility and stability are urgently needed to efficiently convert heat energy into electrical power. Recently, intrinsically crystalline, mechanically stable, and flexible inorganic TE fibers that show TE properties comparable to their bulk counterparts are of interest to researchers. Despite remarkable progress moving TE fibers towards room-temperature TE conversion, the figure-of-merit value $(Z T)$ and bending stability still need enhancement. Herein, we report interfacial engineering enhanced thermoelectric properties of micro-nano polycrystalline TE fibers fabricated by thermally drawing $\mathrm{Bi}_{2} \mathrm{Te}_{3}$-based bulks in a glass-fiber template. The interfacial engineering effect comes from generating stress-induced oriented nanocrystals to increase electrical conductivity and producing strain-distorted interfaces to decrease thermal conductivity. The resulting fibers achieve a $40 \%$ higher $Z T(\sim 1.4$ at $300 \mathrm{~K})$ than their
\end{abstract}


bulk counterparts and show a reversible bending radius of $50 \mu \mathrm{m}$, approaching the theoretical elastic limit. This fabrication strategy works for a wide range of inorganic TE materials and benefits the development of fiber-based micro-TE devices.

\section{Keywords: thermoelectric fibers; $\mathrm{Bi}_{2} \mathrm{Te}_{3}$; thermal drawing; interfacial engineering}

\section{Introduction}

As achieving carbon-dioxide-emission peak and carbon neutrality are significant goals for alleviating global warming and energy problems, an effective solution is to recycle the distributed waste heat energy at medium-or-low temperatures (300-600 K). The capacities of direct conversion between heat energy and electricity have enabled TE devices to achieve power generation and solid-state cooling, in a zero-carbon and sustainable way. ${ }^{1-3}$ The efficiency of TE materials is a key factor for the waste heat energy conversion, which is gauged by its dimensionless figure-of-merit value, $Z T=S^{2} \sigma T / \kappa$, where $S$ is the Seebeck coefficient, $\sigma$ is the electrical conductivity, $T$ is the absolute temperature, and $\kappa$ is the thermal conductivity. However, the $Z T$ value of commercial $\mathrm{Bi}_{2} \mathrm{Te}_{3}$-based materials is restricted to $\sim 1$ at room temperature owing to the adverse interdependence of the Seebeck coefficient, electrical and thermal conductivity. ${ }^{4,5}$ Since $\mathrm{Bi}_{2} \mathrm{Te}_{3}$-based bulk materials with nanostructure have been demonstrated to be one of the best room-temperature TE materials with the highest $Z T \sim 1.4$ at $300 \mathrm{~K}^{6-8}$ they possess large-scale application prospects for TE generators and refrigerators. Moreover, the bulk TE devices are usually made of mechanically cut $\mathrm{Bi}_{2} \mathrm{Te}_{3}$-based pellets, whose elastic strain maxima are experimentally reported to be half lower than the theoretical limit of $5.5 \% .{ }^{9}$ The TE devices can only offer limited flexibility, which makes them difficult to be used on small heat sources with curved shapes. ${ }^{10-12}$ Current solutions mainly focus on embedding/coating the $\mathrm{Bi}_{2} \mathrm{Te}_{3}$-based materials into/on flexible substrates such as yarns, textiles, films, paintings, and papers. ${ }^{13-17}$ However, low TE performance, weak mechanical stability, and complex fabrication process still impede their applications. Hence, simultaneously improving $Z T$ and flexibility of $\mathrm{Bi}_{2} \mathrm{Te}_{3}$-based $\mathrm{TE}$ materials is a great 
challenge, thus it is necessary to explore new strategies to meet the demand.

Over the past 50 years, optical fibers have been an extremely successful platform for optical materials and devices research. The internet and most telecommunication are delivered across the globe today through billions of kilometers of silica fibers, which are typically produced by thermally drawing a preform at the softening temperature of silica glass. ${ }^{18}$ With the same facile technique, multiple materials with disparate properties, including semiconductors, metals, and insulators, can be co-drawn into micro-/nanofibers, which paves the way to a new generation of fibers endowed with unique functionalities at fiber length scales and costs. ${ }^{19,20}$ Based on these studies, ultralong and flexible TE fibers $\left(\mathrm{Bi}_{2} \mathrm{Te}_{3}\right.$, $\mathrm{SnSe}$, etc.) have been fabricated by thermal drawing crystalline TE materials in a glass-fiber preform. ${ }^{21-26} \mathrm{In}$ addition, the TE properties of the $\mathrm{Bi}_{2} \mathrm{Te}_{3} \mathrm{TE}$ fibers can be enhanced due to the preferential orientation of the $\mathrm{Bi}_{2} \mathrm{Te}_{3}$ polycrystalline nanosheets in the core, which were induced by the thermal stress during the fiber drawing process. ${ }^{27}$ However, some disadvantages of the reported TE fibers need to be improved, such as large bending radius, limited $Z T$ value at bulk counterpart level, and low power density for thick glass cladding. On the other hand, the characterization of the comprehensive TE properties (electrical conductivity, Seebeck coefficient, and thermal conductivity) of a single micro-nano fiber is still challenging due to factors such as difficulties in forming good electrical/thermal contact and reducing heat radiation loss. ${ }^{28}$

In this work, we fabricated micro-nano $\mathrm{Bi}_{2} \mathrm{Te}_{3}$-based fibers with diameters ranging from 0.1 to $5 \mu \mathrm{m}$ based on a two-step thermal drawing method. Moreover, a measuring chip was made to characterize the TE properties of fibers and the relative measuring errors were evaluated. Interestingly, we found an interface engineering effect during the fiber drawing process by characterizing the microstructure of microfiber interfaces. The fiber cores were then tuned into oriented nanosheet crystals with rough interfaces, showing increased electrical transport and significantly decreased thermal transport. The $Z T$ value of 4 - $\mu \mathrm{m}$-diameter $\mathrm{Bi}_{2} \mathrm{Te}_{3}$-based fibers was measured to be 1.4 at $300 \mathrm{~K}$, which is $40 \%$ higher than that of their bulk counterpart. Furthermore, the fibers exhibit mechanical stability at a 
curvature radius of $50 \mu \mathrm{m}$, which is three orders of magnitude smaller than the reported inorganic TE fibers, ${ }^{21-24}$ approaching the theoretical limit of $\sim 5.5 \%$ elastic strain for $\mathrm{Bi}_{2} \mathrm{Te}_{3}$ along the $c$ axis at $300 \mathrm{~K}{ }^{9}$ This work benefits the further development of high-performance and highly flexible TE fibers towards widespread applications in energy harvesting and thermal management.

\section{Results and Discussion}

\subsection{Micro-nano thermoelectric fibers fabricated via thermal drawing}

a

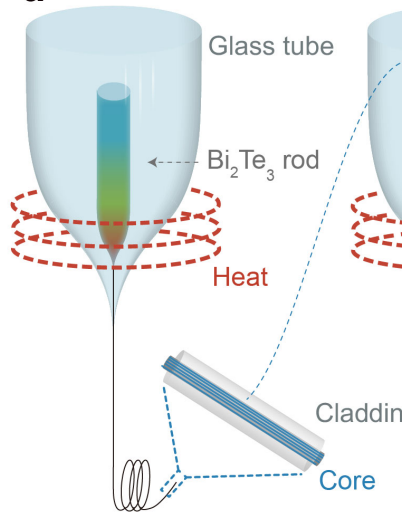

Bulk rod

b

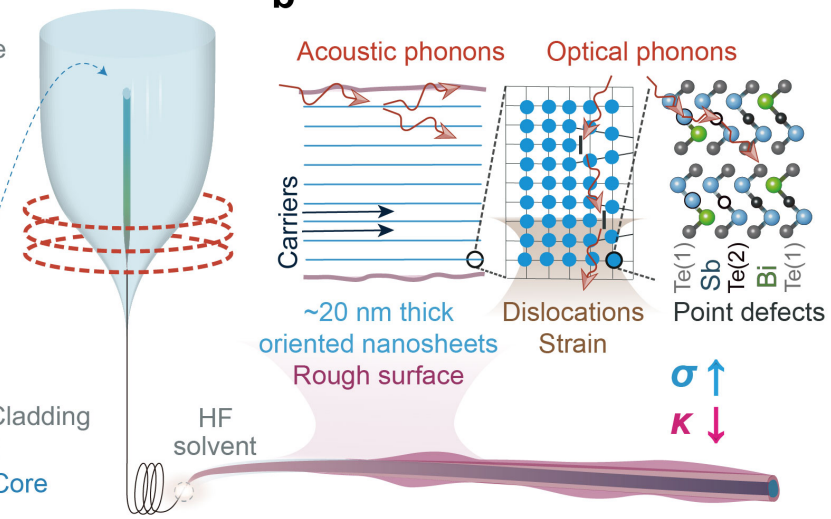

c

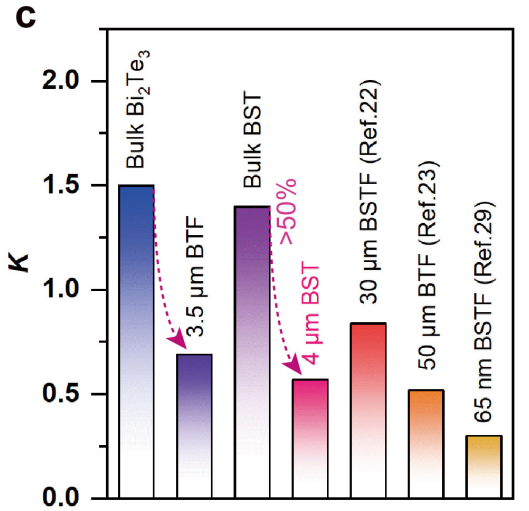

Micro-nano fiber core

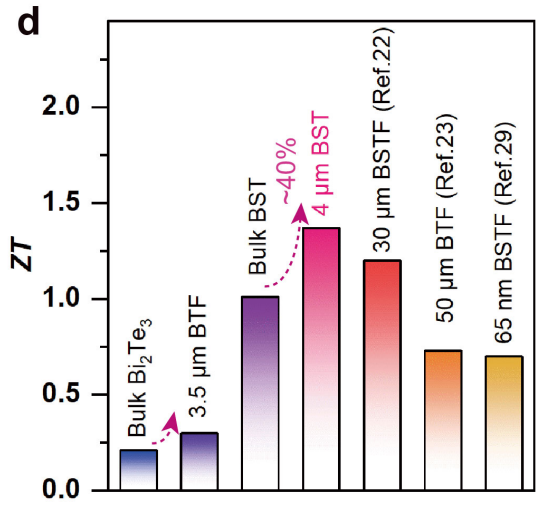

Figure 1. (a) Schematic of micro-nano TE fibers prepared by the glass-fiber-template thermal drawing method. (b) Schematic of the defect structure in the fiber core. (c) Thermal conductivities and (d) $Z T$ values of the p-type $\mathrm{Bi}_{2} \mathrm{Te}_{3} / \mathrm{BST}$ bulks and fibers at $300 \mathrm{~K}$.

Figure 1a shows a schematic of thermal drawing $\mathrm{Bi}_{2} \mathrm{Te}_{3}$-based bulks into fibers. Micro-nano TE fibers with $\mathrm{Bi}_{2} \mathrm{Te}_{3}$ or $\mathrm{Bi}_{0.5} \mathrm{Sb}_{1.5} \mathrm{Te}_{3}$ (BST) core and borosilicate cladding were fabricated by thermal drawing preforms. Especially, the preforms have been endured $700{ }^{\circ} \mathrm{C}$ for a two-hour preheating time before drawing, to form a wetting interface between the core and the cladding by elemental diffusion (see Methods and 
Supplementary Figure S1). The scanning electron microscope (SEM) and energy disperse spectroscope (EDS) were carried out on the micro-nano fiber cross-section with cladding and the hydrogen fluoride (HF) etched fiber core without cladding (Supplementary Figure S2). It shows that the average nanosheet thickness in the fiber core is $\sim 20 \mathrm{~nm}$. Furthermore, the fibers undergo rapid cooling with a rate of $\sim 150^{\circ} \mathrm{C} / \mathrm{s}$ right after thermal drawing, which should cause an interfacial defect as our reported $\mathrm{Bi}_{2} \mathrm{Te}_{3}$ fibers after preheating and fast drawing. ${ }^{23}$ The defect illustration of the fiber cores is exhibited in Figure 1b, which shows that the carrier charges can quickly transport in the oriented nanosheets and multiscale defects can hinder phonon transport.

The TE properties parallel to the fiber-axis direction of the fibers were studied. Figure 1c-1d shows the thermal conductivities $(\kappa)$ and $Z T$ values of the $\mathrm{Bi}_{2} \mathrm{Te}_{3}$ fibers (BTF), $\mathrm{Bi}_{0.5} \mathrm{Sb}_{1.5} \mathrm{Te}_{3}$ fibers (BSTF), and their corresponding bulks. The $\kappa$ of the BTF and BSTF are 50\% lower than that of the corresponding bulks. Importantly, the BSTF with a diameter of $4 \mu \mathrm{m}$ shows an ultra-low $\kappa$ of $0.57 \mathrm{~W} / \mathrm{m} \cdot \mathrm{K}$ and a high $Z T$ of $\sim 1.4$ at $300 \mathrm{~K}$. The micro-nano fiber without glass cladding should possess a higher output power density than the reported $\mathrm{mW} / \mathrm{cm}^{2}$ level of TE fibers with glass cladding. ${ }^{22}$ To highlight the advantages of the micro-nano TE fibers, the thermal conductivities and the $Z T$ values of other state-of-the-art $\mathrm{Bi}_{2} \mathrm{Te}_{3}$-based fibers are also compared in Figure 1c-1d. ${ }^{22,23,29}$ The enhanced TE properties and the corresponding mechanism of the interfacial engineering effect will be discussed as follows.

\subsection{Interfacial microstructure}



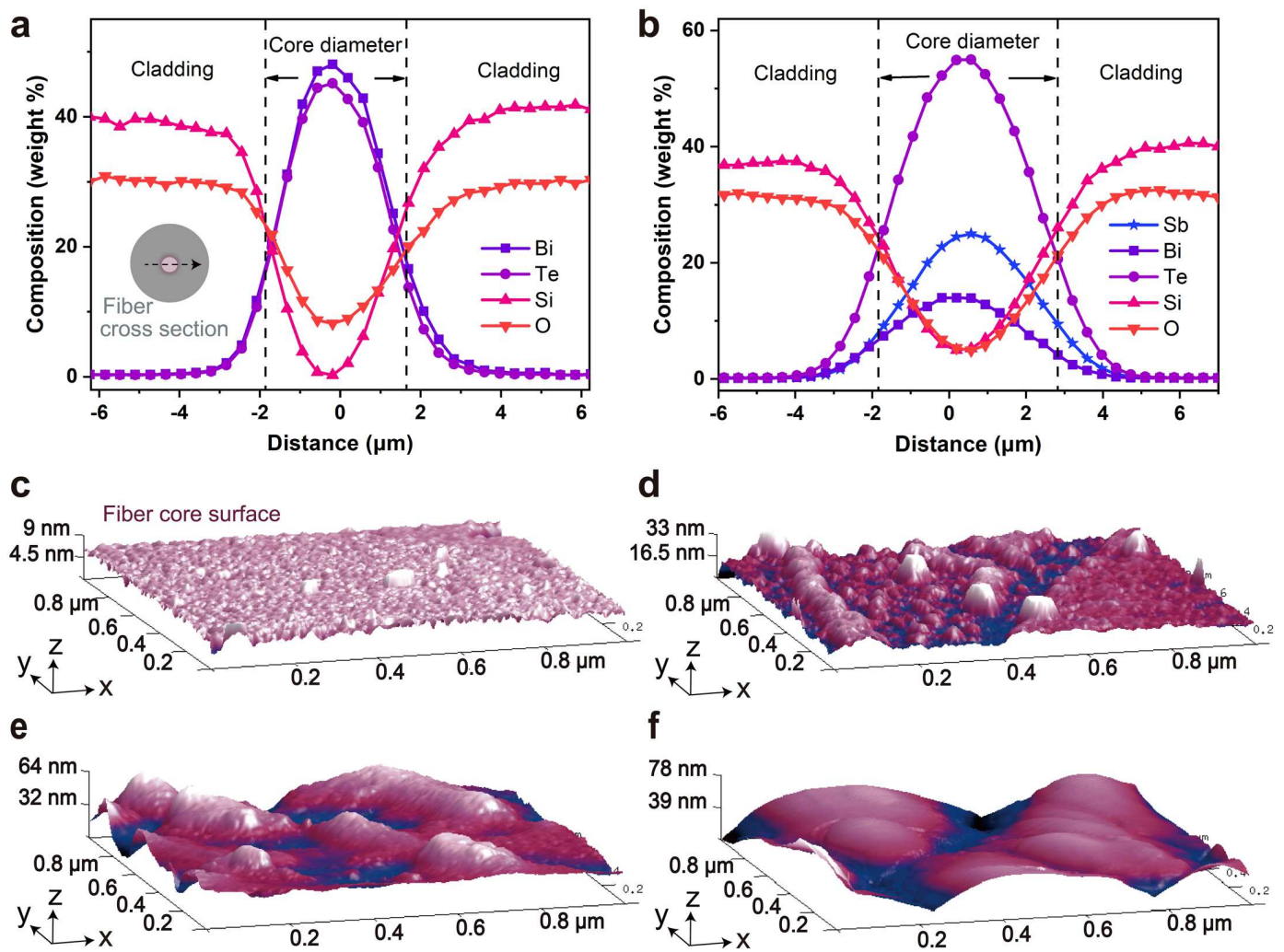

Figure 2. Linear elemental analysis on the cross-section of (a) BTF and (b) BSTF. Three dimensional AFM images of the surfaces of the BSTF cores with different diameters: (c) $100 \mu \mathrm{m}$; (d) $20 \mu \mathrm{m}$; (e) $5 \mu \mathrm{m}$; (f) $1 \mu \mathrm{m}$.

To understand the mechanism of the ultra-low thermal conductivities and high electrical conductivities in the micro-nano fibers, further analysis was performed on the microfiber by electro-probe microanalyzer (EPMA), atomic force microscopy (AFM), and transmission electron microscopy (TEM). The detailed information can be found in Methods. Figure 2a-2b exhibits the linear elemental (Bi, Sb, Te, Si, and O) profiles across the polished interface of the BTF and BSTF by EPMA measurement. There exists little diffusion of $\mathrm{Bi}, \mathrm{Sb}$, and $\mathrm{Te}$ into the cladding, while there is some diffusion of $\mathrm{Si}$ and $\mathrm{O}$ into the core region. The diffused elements should form some nanoscale oxides in the core, such as $\mathrm{SiO}_{2}$ and $\mathrm{TeO}_{2}$, which will have strong phonons scattering, resulting in lower thermal conductivity as in our previous studies. ${ }^{23}$ The elemental diffusion introduces an oxidic surface of the fiber core, which could protect the fiber core from atmospheric oxidation.

Figure $2 \mathrm{c}-2 \mathrm{f}$ shows the surface roughness of the BSTF cores with diameters of $100,20,5$, and $1 \mu \mathrm{m}$, respectively. When the diameter is $100 \mu \mathrm{m}$, the surface of the as-prepared fiber core is relatively flat, however, it becomes rougher as the core 
diameter decreases. When the diameter is $1 \mu \mathrm{m}$, the height of the humps dramatically increases. The surface roughness was measured to be $9,33,64$, and $78 \mathrm{~nm}$, corresponding to the fiber diameters of $100,20,5$, and $1 \mu \mathrm{m}$, respectively. The enhancement in the surface roughness with the decreasing core diameter could be attributed to the increased depth of elemental diffusion as mentioned above. Notably, besides the well-recognized classical size effect due to the phonon boundary scattering, the surface roughness can also significantly alter the thermal conductivity. ${ }^{30}$

\section{a}

(i)
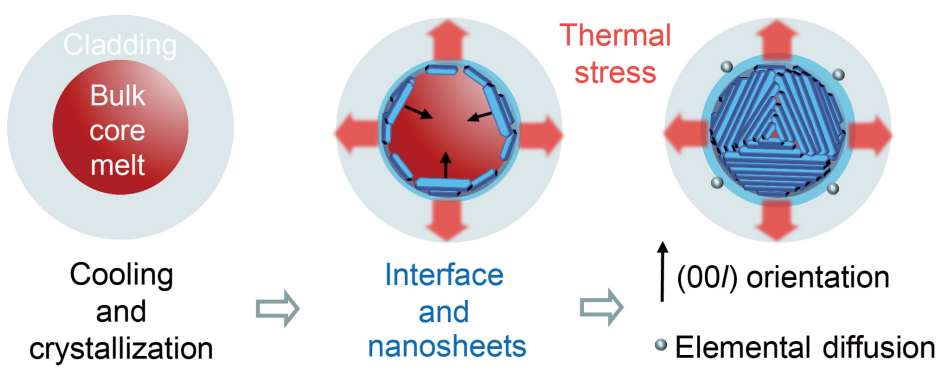

(ii)
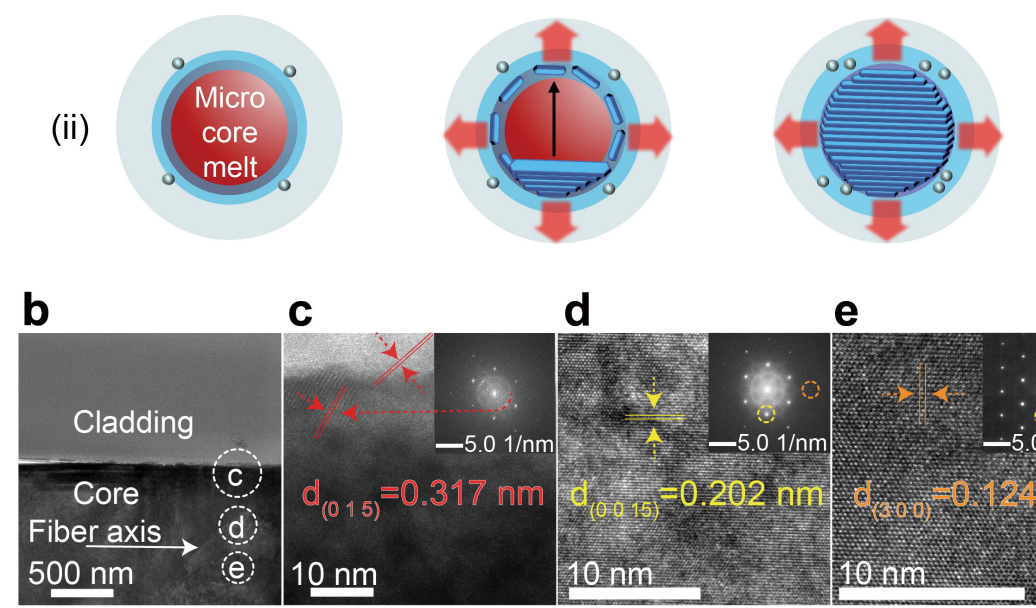

\section{d}

e

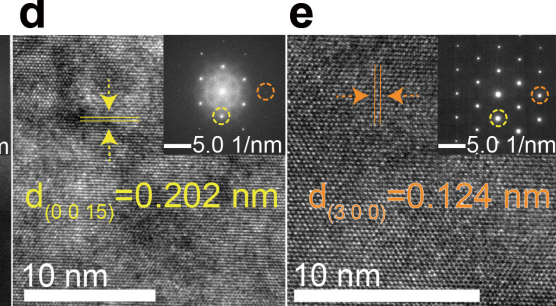

Figure 3. (a) Oriented crystal growth model of the $\mathrm{Bi}_{2} \mathrm{Te}_{3}$ fiber core via thermal drawing. (b) HAADF image of a BSTF chip and (c)-(e) enlarged view of the circled region in (b). The insets of (c)-(e) are corresponding SAED patterns.

To reveal the growth process of the oriented nanosheet crystal in the fiber confined field, which is shown in Figure 1 and Supplementary Figure S2, the morphology evolution during the fiber-drawing process is schematically shown in Figure 3a. During the cooling process of the first thermal drawing (part i), the $\mathrm{Bi}_{2} \mathrm{Te}_{3}$ melt gradually nucleates and grows into hexagonal nanosheets around the interface to minimize potential energy. The nanosheet crystals will continuously stack around the interface and then the crystals grow along the $\left(\begin{array}{lll}0 & 0 l\end{array}\right)$ plane and compete in several 
directions into a flower shape (Supplementary Figure S2a). More interestingly, during the second drawing process (part ii), the nanosheet crystals stack around the interface at the beginning. Accompanied by smaller fiber-core space and larger interface stress than the first drawing process, then the crystals preferentially grow along the $\left(\begin{array}{lll}0 & 0 & l\end{array}\right)$ plane in one direction (Supplementary Figure S2b). Meanwhile, elemental diffusion takes place between the cladding and the core, which causes an increase in the surface roughness of the fiber core as found above.

Figure $3 \mathrm{~b}$ presents a HAADF image of a BSTF sample processed by using a focused ion beam (FIB) method, including the upside core and the underside cladding of the fiber. Figures 3c-3e show the enlarged views of the circled region in Figure $3 b$, from the interface to the core of $0,0.5$, and $1 \mu \mathrm{m}$ depth. The insects of Figures $3 \mathrm{c}-3 \mathrm{e}$ present the corresponding images of selected area electron diffraction (SAED). These measurements confirm the crystalline state of $\mathrm{Bi}_{0.5} \mathrm{Sb}_{1.5} \mathrm{Te}_{3}$ in the fiber core. Figure $3 \mathrm{c}$ shows that the core nearby the interface is polycrystalline with a lattice-plane spacing of $0.317 \mathrm{~nm}$, corresponding to the $\left(\begin{array}{lll}0 & 1 & 5\end{array}\right)$ plane. It is noted that the core in $1 \mu \mathrm{m}$ depth away from the interface is an ordered polycrystalline state, as shown in Fig. 3d. In addition, it includes diverse dislocations and strain distortions (Supplementary Figure $\mathrm{S} 3$ ), which is similar to the reported mosaic crystal. ${ }^{31}$ And the insert of Figure $3 \mathrm{~d}$ marks the corresponding lattice planes, including strong $\left(\begin{array}{lll}0 & 0 & 15\end{array}\right)$ and weak $\left(\begin{array}{lll}3 & 0 & 0\end{array}\right)$.

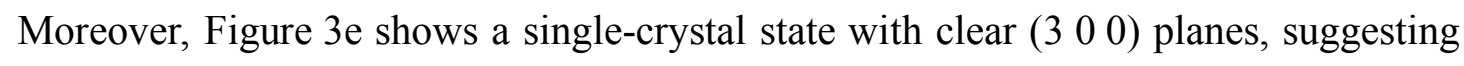
the direction of the $\mathrm{Bi}_{0.5} \mathrm{Sb}_{1.5} \mathrm{Te}_{3}\left(\begin{array}{lll}3 & 0 & 0\end{array}\right)$ planes are parallel to the fiber axis. These results verify the effect of interfacial engineering on the ordered and defective microstructure of the fiber core (Figure 1b), which will enhance the electrical transport rather than the thermal transport. ${ }^{32}$ As a result, the fiber cores could exhibit high $\mu / \kappa_{l}$ ( $\mu$ is the carrier mobility and $\kappa_{l}$ is the lattice thermal conductivity), which is proportional to the $Z T$ value for high TE performance evaluation. ${ }^{33}$

\subsection{Measured room-temperature thermoelectric properties}



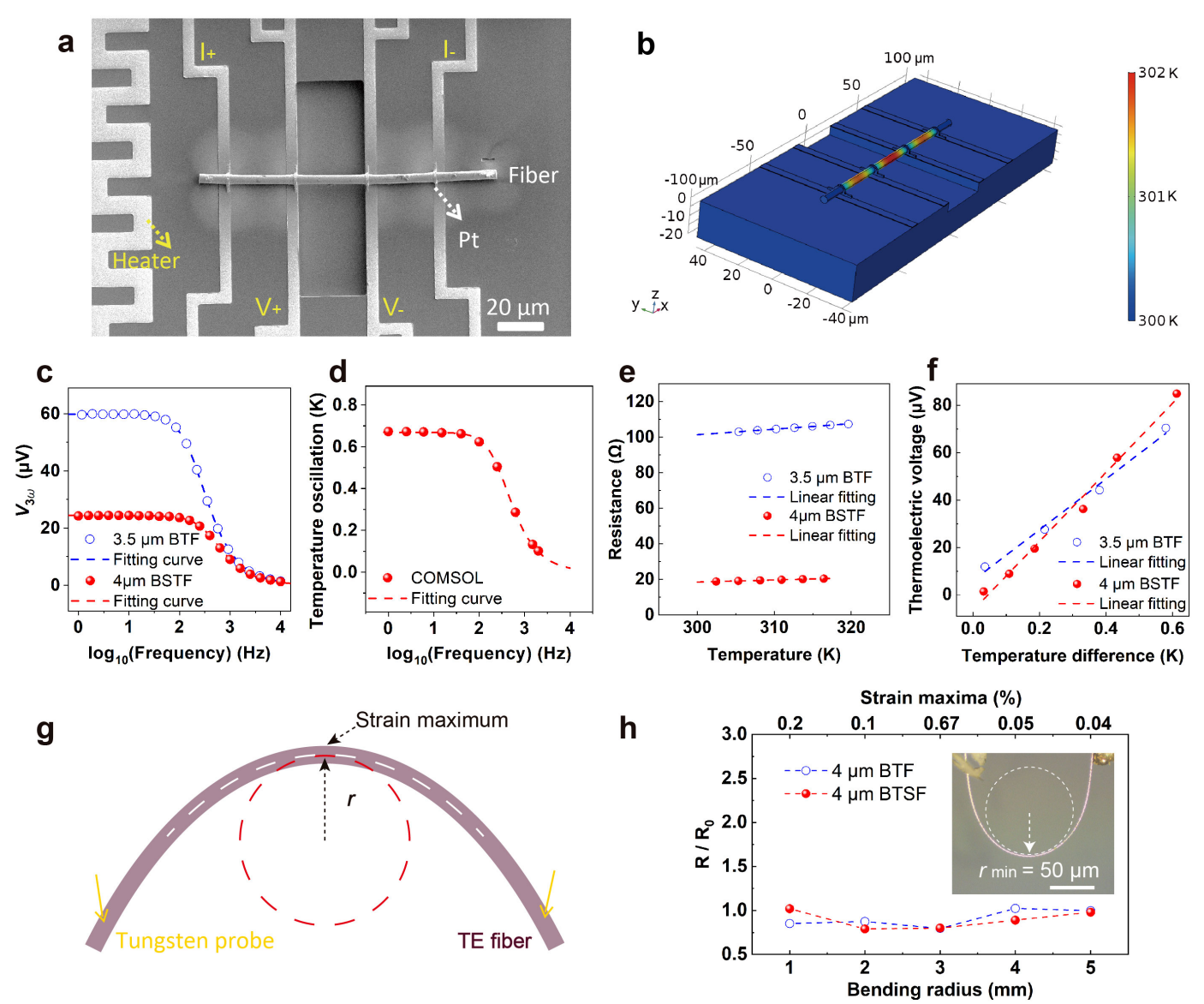

Figure 4. (a) SEM image of the BSTF bonded on the Si test chip. (b) FEM model for simulating the data-reduction process of the self-heating $3 \omega$ method with COMSOL software. (c)

Frequency-dependent $V_{3 \omega}$ values measured from two fibers and (d) peak amplitude of the temperature oscillation calculated from COMSOL for a $4-\mu \mathrm{m}$-diameter fiber. (e)

Temperature-dependent electrical resistance and (f) temperature-difference-dependent thermoelectric voltage. (g) Schematic of the fiber bending model. (h) Bending-radius-dependent relative electrical resistance; $R$ and $R_{0}$ are the resistances in the bending state and original flat state, respectively; Inset: an optical microscopic image of a bent BSTF.

The electrical conductivity $(\sigma)$, Seebeck coefficient $(S)$, and thermal conductivity $(\kappa)$ of the fiber were measured by using a pre-patterned silicon (Si) chip. Therefore the TE properties can be in-situ measured from one fiber. As shown in Figure $4 \mathrm{a}, \mathrm{a}_{2} \mathrm{Bi}_{3}$ fiber is placed over four golden electrodes for the thermal conductivity and electrical conductivity measurements on the test chip, in which two outer ones ( $\mathrm{I}_{+}$and $\left.\mathrm{I}_{-}\right)$and two inner ones $\left(\mathrm{V}_{+}\right.$and $\left.\mathrm{V}_{-}\right)$are used for sourcing current and extracting voltage, respectively. One end of the fiber is located close to a golden heater which is used to create a temperature gradient for the $S$ measurement. Once the heater was turned on, the two inner electrodes were also used as thermometers to probe the temperature 
difference across the central portion of the fiber. The bonding between the fiber and the electrodes was created by depositing platinum (Pt) by applying FIB. The chip was subsequently annealed at $40^{\circ} \mathrm{C}$ for 10 minutes to cure the welding spot of Pt. The axial thermal conductivity $\kappa_{\|}$of the suspended fiber was measured by the self-heating $3 \omega$ method. ${ }^{28}$ In this method, the fiber is periodically heated by an alternating current (AC) at an angular frequency $\omega$. The magnitude of the average temperature oscillation of the central portion of the fiber is proportional to a $3 \omega$ voltage:

$$
\left|V_{3 \omega}\right|_{r m s}=\frac{4 I_{r m s}^{3} L R_{s}^{2} \beta_{s}}{\pi^{4} \kappa_{\mathrm{P}} A_{s} \sqrt{1+\left(2 \omega \gamma_{t}\right)^{2}}}
$$

where $\left|V_{3 \omega}\right|_{r m s}$ and $I_{r m s}$ are the root-mean-square amplitude of the $3 \omega$ voltage and the exciting current, respectively. $L$ is the length of the central portion of the fiber. $R_{s}, \beta_{s}$, and $A_{s}$ are the resistance, temperature coefficient of the resistance (TCR), and the cross-sectional area, respectively. $\gamma_{t}$ represents a characteristic thermal time constant associated with the heat transfer along the fiber axial direction. The measured $\left|V_{3 \omega}\right|_{r m s}$ data can be used to fit Equation (1) to extract the axial thermal conductivity $\kappa_{\|}$. Notice that a $30 \mu \mathrm{m}$ wide trench is created between the two inner electrodes $\left(\mathrm{V}_{+}\right.$and $\left.\mathrm{V}_{-}\right)$on the silicon substrate to make the central portion of the fiber (sensing region) suspended. This will eliminate the heat flow between the sensing region and the substrate, which is necessary for properly implementing the self-heating $3 \omega$ method. Figure 4c shows the experimentally measured $\left|V_{3 \omega}\right|_{r m s}$ values of the two fibers as a function of exciting current frequency $f=\omega / 2 \pi$. The obtained $\kappa \|$ of 3.5 - $\mu$ m-diameter BTF and $4-\mu \mathrm{m}$-diameter BSTF are $0.69 \pm 0.06$ and $0.57 \pm 0.05 \mathrm{~W} / \mathrm{m} \cdot \mathrm{K}$, respectively.

The reliability of the current experimental configuration for measuring $\kappa_{\|}$could be affected by the surface radiation of the fiber and the thermal contact of Pt bonding. To address these issues, we have applied the $\mathrm{COMSOL}^{\mathrm{TM}}$ software to perform a finite element modeling (FEM) calculation on a $4-\mu$ m-diameter fiber with a pre-set $\kappa_{\|}$value of $0.5 \mathrm{~W} / \mathrm{m} \cdot \mathrm{K}$ (Figure $4 \mathrm{~b}$ ). In the simulation, we assigned on purpose a unity emissivity to the surfaces of the fiber and Pt bonding. The thermophysical properties 
and experimental dimension of the Pt bonding were also carefully considered. Figure $4 \mathrm{~d}$ shows the theoretically predicted peak amplitude of temperature oscillation for the 4- $\mu$ m-diameter fiber. By performing a similar nonlinear fitting on the data shown in Figure $4 \mathrm{~d}$ (see Methods), the $\kappa_{\|}$derived from the FEM simulation is found to be $0.455 \mathrm{~W} / \mathrm{m} \cdot \mathrm{K}$, which is about $9 \%$ smaller than its pre-set value. Thus, we believe that the $3 \omega$ measurements based on the current configuration are reliable. The temperature-dependent electrical resistances of two fibers were measured by the four-probe method, from which the TCRs in Equation (1) and the $\sigma$ can be calculated (Figure 4e). The $S$ of the two fibers were measured by using a steady-state method. ${ }^{34}$ In this method, the heater was powered by a DC current source, which can create temperature gradients of different magnitude across the fiber, and the corresponding TE voltages $(\mathrm{d} V)$ were measured by a Keithley 2400 soucemeter (Figure $4 \mathrm{f}$ ). The $S$ can be calculated from the slope of the $\mathrm{d} V-\mathrm{d} T$ linear fitting. Detailed descriptions of the experimental implementation and simulation of the self-heating $3 \omega$ method can be found in Methods.

The obtained TE properties of the fiber samples are listed in Table 1 with measuring uncertainties (Supplementary Note 1) in comparison with those reported in the literature. Except for $S$, the values of $\sigma, \kappa_{\|}$, and $Z T$ of the prepared fibers are all improved with respect to those of bulk materials at different levels. Remarkably, the fiber core exhibits an ultralow $\kappa$ and enhanced $\sigma$, which are mainly contributed to the interfacial defect structure and inner-oriented nanosheet crystals. The nanosheet thickness of $\sim 20 \mathrm{~nm}$ is close to the wavelength of phonons and larger than that of majority carriers, so the nanosheet boundaries could selectively scatter phonons and make carriers pass through. ${ }^{35}$ The phonon modulation is then investigated by Vienna $\mathrm{Ab}$ initio Simulation Package (VASP) software based on the Density Functional Theory (DFT). The calculation details can be found in Supplementary Note 2. The study confirms the effect of different mean-free-path phonons on the lattice thermal conductivity of $\mathrm{Bi}_{2} \mathrm{Te}_{3}$ at $300 \mathrm{~K}$ (Supplementary Figure S5). What's more, the nanostructure of $\mathrm{Bi}_{2} \mathrm{Te}_{3}$ under $20 \mathrm{~nm}$ could efficiently scatter phonons and depress the lattice thermal conductivity at $300 \mathrm{~K}$. 
The fiber core without cladding can keep $90 \%$ of its original electrical conductivity value after being preserved in the atmosphere for more than one year. (Supplementary Figure S6). And these fibers do not require using ion milling/etching as reported for nanowires to cleave surface oxides and measure TE properties. ${ }^{29,36}$ Beyond these, we carry out a bending procedure and consecutively measure I-V characteristics of the representative BTF and BSTF (fiber diameter $D \sim 4 \mu \mathrm{m}$ ) by applying two gold-plated tungsten probes (Figure 4g and Supplementary Figure S7). Using the original resistance $R_{0}$ as a reference, the bending-state resistance $R$ is normalized to exhibit a change of the electrical resistance as a function of $1-5 \mathrm{~mm}$ bending radius $r$ (Figure 4h). After exerting 100-time bending cycles at $r \sim 5 \mathrm{~mm}$, the resistance increases less than 5\%, indicating its tremendous mechanical flexibility (Supplementary Figure S7 and Supplementary Movie 1). Meanwhile, the bending radius minima $\left(r_{\min }\right)$ of all referred samples are also listed in Table 1. Their flexibility can also be estimated by the maximum bending strain $(\varepsilon=D / 2 r) \cdot{ }^{37}$ It exhibits that these microfibers possess $r_{\min } \sim 50 \mu \mathrm{m}$ an excellent $\varepsilon$ of $4 \%$, approaching that of the reported nanowires and the $\sim 5.5 \%$ theoretical limit of $\mathrm{Bi}_{2} \mathrm{Te}_{3}$ elastic strain. ${ }^{9,38}$ These results demonstrate that the thermally drawn micro-nano $\mathrm{Bi}_{2} \mathrm{Te}_{3}$ fibers with interfacial engineering effects possess both high TE performance and great bending stability.

Table1. Thermoelectric and bending properties of $\mathrm{Bi}_{2} \mathrm{Te}_{3}$ bulk and fiber samples

\begin{tabular}{|c|c|c|c|c|c|}
\hline $\begin{array}{l}\text { Samples } \\
\text { at } 300 \mathrm{~K}\end{array}$ & $\begin{array}{r}S \\
(\mu \mathrm{V} / \mathrm{K})\end{array}$ & $\begin{array}{c}\sigma \\
\left(10^{4} \mathrm{~S} / \mathrm{m}\right)\end{array}$ & $\begin{array}{c}\kappa_{\|} \\
(\mathrm{W} / \mathrm{m} \cdot \mathrm{K})\end{array}$ & $Z T$ & $r_{\min }$ \\
\hline Bulk $\mathrm{Bi}_{2} \mathrm{Te}_{3}$ & 114 & 5.0 & 1.50 & 0.21 & / \\
\hline $3.5 \mu \mathrm{m} \mathrm{BTF}$ & $107 \pm 12$ & $5.9 \pm 0.3$ & $0.69 \pm 0.06$ & $0.3 \pm 0.07$ & $45 \pm 5 \mu \mathrm{m}$ \\
\hline Bulk BST & 212 & 10.5 & 1.4 & 1.01 & l \\
\hline $4 \mu \mathrm{m}$ BSTF & $145 \pm 16$ & $12.5 \pm 0.7$ & $0.57 \pm 0.05$ & $1.38 \pm 0.34$ & $50 \pm 5 \mu \mathrm{m}$ \\
\hline $30 \mu \mathrm{mST}^{22}$ & 150 & 15.6 & 0.84 & 1.25 & $1 \mathrm{~cm}$ \\
\hline $50 \mu \mathrm{m} \mathrm{Bi}_{2} \mathrm{Te}_{3}{ }^{23}$ & 131 & 7.4 & 0.52 & 0.73 & $5 \mathrm{~cm}$ \\
\hline $65 \mathrm{~nm} \mathrm{BST}^{29}$ & 125 & 4.5 & 0.3 & 0.7 & $500 \mathrm{~nm}$ \\
\hline
\end{tabular}




\section{Conclusions}

In conclusion, we have demonstrated the high-performance micro-nano polycrystalline $\mathrm{Bi}_{2} \mathrm{Te}_{3} \mathrm{TE}$ fibers with great flexibility and stability through thermal drawing $\mathrm{Bi}_{2} \mathrm{Te}_{3}$-based bulks in a glass-fiber template. A special interface between the glass cladding and the $\mathrm{Bi}_{2} \mathrm{Te}_{3}$ core was observed to induce oriented nanosheet crystals in the fiber cores, enabling the fibers to display ultra-low thermal conductivity and high electrical conductivity. Furthermore, this interfacial engineering efficiently regulates the electrical and thermal transport, while keeping the electrical properties stably after 100-time 5-mm-diameter bending and long-time preservation. This strategy could be universal to other inorganic TE materials, particularly those made from nontoxic and abundant elements. The high TE performance and stability in the micro-nano TE fibers will stimulate further research on the TE fiber materials (e.g. fibrous crystal anisotropy and quantum size effect) and the development of integrated TE fiber devices (e.g. self-powered wearable electronics and micro-area temperature management).

\section{Methods}

4.1 Fabrication of micro-nano TE fibers. The glass-fiber-template method and two-step thermal drawing were applied for micro-nano $\mathrm{Bi}_{2} \mathrm{Te}_{3}$ fibers fabrication. First, a 10-mm-diameter $\mathrm{Bi}_{2} \mathrm{Te}_{3}$ or $\mathrm{Bi}_{0.5} \mathrm{Sb}_{1.5} \mathrm{Te}_{3} \operatorname{rod}(99.99 \%$ purity, Santech Materials, Changsha) was sealed in a Pyrex3.3-borosilicate-glass cladding to assemble a 30-mm-diameter macroscopic cylindrical preform. The preforms were preheated at $700{ }^{\circ} \mathrm{C}$ for two-hour before drawing to form a wetting interface of the core and the cladding by elemental diffusion. The TE fibers were then drawn at $\sim 950{ }^{\circ} \mathrm{C}$ using an optical fiber drawing tower. The fibers endure flash cooling with a rate of $\sim 150{ }^{\circ} \mathrm{C} / \mathrm{s}$ right after thermal drawing, but all fibers maintain a complete core-cladding structure and the fiber cores show a relative density of $>99.2 \%$. The $400-\mu \mathrm{m}$-diameter $\mathrm{Bi}_{2} \mathrm{Te}_{3}$-based fiber prepared by the first thermal drawing was then inserted into a glass tube of Pyrex3.3 borosilicate to form a new fiber preform. Cascading two thermal 
drawing steps, continuous meters of micro-nano $\mathrm{Bi}_{2} \mathrm{Te}_{3}$-based $\mathrm{TE}$ fibers were produced.

4.2 Composition and microstructure characterizations. Utilizing an argon ion beam cross-section polisher (CP, JEOL IB-09020CP, Japan), the as-drawn fibers were polished to obtain a cross-sectional sample and a longitudinal section sample. And the two fiber samples were observed under a field electron-scanning electron microscope (ZEISS Merlin, FE-SEM, Germany) operated at $20 \mathrm{kV}$ with a working distance of 8.4 mm. The $\mathrm{Bi}_{2} \mathrm{Te}_{3}$ core was obtained by etching the fibers in HF acid solution to strip the glass cladding and then identified by an X-ray diffractometer (XRD, X'Pert PROX, $\mathrm{Cu} \mathrm{Ka}$, Netherland). Using an electron probe micro-analyzer (Shimadzu EPMA-1600, Japan) equipped with a wavelength-dispersive spectrometer, elemental and microstructural analyses were performed on the fibers at several locations traversing the core in approximately $1 \mu \mathrm{m}$ increments to examine the distribution of elements. A chip sample, containing the fiber core/cladding interface region of the fiber, was cut out from the fiber by a focused gallium ion beam instrument (FIB, FEI Helios 450S, America). High-resolution transmission electron microscopy (HR-TEM) observations on this chip sample were carried out at $200 \mathrm{kV}$ using a transmission electron microscope (FEI Titan Themis 200, X-FEG TEM).

4.3 Self-heating $3 \omega$ measurement. The thermal conductivity $\kappa_{\|}$and volumetric heat capacity $C_{V}$ of the suspended $\mathrm{Bi}_{2} \mathrm{Te}_{3}$ fiber were measured by a self-heating $3 \omega$ method proposed originally by Lv and co-workers. In this method, the electrically conductive sample acts as not only a heater but also a resistive temperature sensor, since it owns a substantially large temperature coefficient of resistance (TCR). Figure S4 shows a schematic of the electrical circuit for carrying out the $3 \omega$ measurement on the fiber integrated test chip using a four-probe configuration. ${ }^{39,40}$ (To reduce the convective heat loss, the test chip was installed inside a vacuum chamber evacuated to below $5 \times 10^{-4} \mathrm{~Pa}$ during the measurement). A sinusoidal current at frequency $\omega$ supplied by a Keithley 6221 current source was injected into the fiber from two outer contacts $\left(\mathrm{I}_{+}\right.$ and I.) on the test chip. The periodic heating induced a resistance fluctuation of the fiber at frequency $2 \omega$. Multiplication of the $2 \omega$ resistance variation by the $1 \omega$ driving 
current gives rise to a small $3 \omega$ voltage which can be used to extract the thermo-physical properties of the sample by using Equation (1).

The voltage extracted from two inner contacts $\left(\mathrm{V}_{+}\right.$and $\left.\mathrm{V}_{-}\right)$contain both $1 \omega$ component $\left(V_{1 \omega}\right)$ and $3 \omega$ component $\left(V_{3 \omega}\right)$. Since the amplitude of $V_{1 \omega}$ is usually $3-4$ orders of magnitude larger than that of $V_{3 \omega}, V_{1 \omega}$ should be filtered out to achieve faithfully recording of the $3 \omega$ voltage signal. Hence the fiber was electrically connected with a potentiometer in series. The resistance of the potentiometer was adjusted to be slightly larger than that of the fiber. After passing through a differential amplifier, the voltage signal extracted from the fiber was fed into the input A of a Stanford SR850 lock-in amplifier, while the $1 \omega$ voltage signal $V_{1 \omega, \mathrm{P}}$ from the potentiometer was passed to a 12-bit DAC converter in which the amplitude of $V_{1 \omega, \mathrm{P}}$ was adjusted to be equal to that of $V_{1 \omega}$ by controlling the gain of the DAC with a computer. The attenuated $V_{1 \omega, \mathrm{P}}$ signal was then sent to the input $\mathrm{B}$ of the lock-in amplifier to deduct out $V_{1 \omega}$ by using a differential input mode. After the signal subtraction step, $V_{3 \omega}$ can be measured by the same lock-in amplifier.

4.4 Finite element simulation of $3 \omega$ measurement. A 3D FEM simulation has been carried out to evaluate the reliability of measuring the thermal conductivity of the fiber on the test chip with the geometrical configuration shown in Figure 4a. A simulation model was constructed to imitate the chip setup, in which the diameter and length of the suspended portion of the fiber were $4 \mu \mathrm{m}$ and $30 \mu \mathrm{m}$, respectively. (Figure 4b) The fiber was assigned a thermal conductivity of $0.5 \mathrm{~W} / \mathrm{m} \cdot \mathrm{K}$ and a volumetric heat capacity of $1.22 \times 10^{6} \mathrm{~J} /\left(\mathrm{m}^{3} \mathrm{~K}\right) .{ }^{41}$ Lee and co-workers found that the platinum film created by the FIB deposition mainly consists of carbon $(45 \%-55 \%)$ and platinum $(40 \%-50 \%){ }^{42}$ The thermal conductivity of the $\mathrm{Pt} / \mathrm{C}$ film was measured to be $40.97 \mathrm{~W} / \mathrm{m} \cdot \mathrm{K}$. In the simulation, the thermal conductivity of the Pt bonding was set to be $40 \mathrm{~W} / \mathrm{m} \cdot \mathrm{K}$. We further assumed that the $\mathrm{Pt} / \mathrm{C}$ bonding is composed of $50 \%$ amorphous carbon and $50 \%$ platinum in volume, ${ }^{42}$ therefore the volumetric heat capacity of the $\mathrm{Pt} / \mathrm{C}$ bonding was approximated to be $2.98 \times 10^{6} \mathrm{~J} /\left(\mathrm{m}^{3} \mathrm{~K}\right)$. From Lee's work, ${ }^{43}$ we also estimated that the electrical conductivity of the $\mathrm{Pt} / \mathrm{C}$ material is about 72.04 S/m. To initiate the simulation, an AC electrical current with a peak amplitude 
$I_{0}$ of $0.4 \mathrm{~mA}$ and an angular frequency of $\omega$ was injected into the fiber from two outer electrodes to create periodical heating at frequency $2 \omega$ in vacuum at room temperature. The temperature distribution of the test chip was calculated by coupling the heat transfer module with the AC/DC module implemented in the COMSOL ${ }^{\mathrm{TM}}$ software. The surface heat radiation loss was modeled by employing the Stefan-Boltzmann law. The material properties of the fiber, $\mathrm{Pt} / \mathrm{C}$ bonding, gold strips ${ }^{44,45}$ and silicon substrate used in the FEM modeling are summarized in Table 2, which are all assumed to be temperature independent.

Table 2. Materials properties used in the FEM simulation

\begin{tabular}{ccccc}
\hline \hline & $\mathrm{Bi}_{2} \mathrm{Te}_{3}{ }^{41}$ & $\mathrm{Pt} / \mathrm{C}^{43}$ & $\mathrm{Au}^{44}$ & $\mathrm{Si}^{45}$ \\
\hline $\begin{array}{c}\kappa \\
\left(\mathrm{W} \mathrm{m}^{-1} \mathrm{~K}^{-1}\right)\end{array}$ & 0.5 & 40 & 240.00 & 142.2 \\
$\begin{array}{c}C_{V} \\
\left(\mathrm{~J} \mathrm{~m}^{-3} \mathrm{~K}^{-1}\right)\end{array}$ & $1.22 \times 10^{6}$ & $2.98 \times 10^{6}$ & $1.60 \times 10^{6}$ & $1.63 \times 10^{6}$ \\
$\gamma$ & 1 & 1 & 0.03 & 0.5 \\
$\sigma$ & $1.23 \times 10^{5}$ & 72.04 & $5.23 \times 10^{6}$ & $/$ \\
\hline \hline
\end{tabular}

Supplementary Figure S7 illustrates a typical time-dependent spatially averaged temperature oscillation curve extracted from the central portion of the fiber heated by the electrical current at $f=15 \mathrm{~Hz} \quad(f=\omega / 2 \pi)$. The peak amplitude $\left|\Delta T_{2 \omega}\right|_{p}$ of the temperature oscillation can be displayed as a function of exciting current frequency, as shown in Figure 4d. Notice that in the real experiment $\left|\Delta T_{2 \omega}\right|_{p}$ can be derived from the root-mean-square amplitude of the $3 \omega$ voltage $\left|V_{3 \omega}\right|_{r m s}$ by:

$$
\left|V_{3 \omega}\right|_{r m s}=\frac{I_{r m s} R_{s} \beta_{s}\left|\Delta T_{2 \omega}\right|_{p}}{2}
$$

where $I_{\text {rms }}$ is the root-mean-square amplitude of the exciting current. $R_{s}$ is the resistance of the suspended portion of the fiber. $\beta_{s}$ is the TCR of the fiber. Substituting Equation (2) into Equation (1) yields temperature oscillation: 


$$
\left|\Delta T_{2 \omega}\right|_{p}=\frac{8 I_{r m s}^{2} R_{s} L}{\pi^{4} \kappa_{\mathrm{P}} A_{s} \sqrt{1+\left(2 \omega \gamma_{\mathrm{t}}\right)^{2}}}
$$

where $A_{S}$ is the cross-sectional area of the fiber. By fitting the $\left|\Delta T_{2 \omega}\right|_{p}$ vs. $f$ curve in Figure $4 \mathrm{~d}$ to Equation (3), the thermal conductivity was found to be $0.455 \mathrm{~W} /(\mathrm{mK})$.

\section{Acknowledgments}

The authors acknowledge funding from the Natural Science Foundation of China (52002131, U1830203, 62005080, 52172249, 51888103, 91833303), 2021 Talent Revitalization Plan Project for New High Performance Material Industry in Qingyuan City (2021YFJH02001), Local Innovative and Research Teams Project of Guangdong Pearl River Talents Program (2017BT01X137), Key R\&D Program of Guangzhou (202007020003), and Innovation Academy for Light-duty Gas Turbine, Chinese Academy of Sciences (CXYJJ21-ZD-02). The authors acknowledge Dr. Ben Huang and Prof. Pengcheng Zhai at Wuhan University of Technology for providing mechanical discussions of thermoelectric fibers.

\section{Author contributions}

M.S., G.W.T., P.Y.Z., and B.H. prepared the micro-nano TE fibers and conducted EPMA, AFM, and TEM characterization. M.S., G.W.T., and H.F.W. performed TE properties measurements and wrote the original manuscript. M.Y., H.Z., and T.Z. performed theoretical simulation. M.S., Y.C.C., and J.C. conducted stability tests of electrical performance. M.S., G.W.T., H.F.W., D.D.C., and J.L.G. analyzed results. Q.Q. and Z.M.Y. directed this project.

\section{References}

1 Snyder, G. J. \& Toberer, E. S. Complex thermoelectric materials. Nature Materials 7, 105-114, doi:10.1038/nmat2090 (2008).

2 Tarancon, A. Powering the IoT revolution with heat. Nat Electron 2, 270-271, doi:10.1038/s41928-019-0276-4 (2019).

3 Geffroy, C., Lilley, D., Parez, P. S. \& Prasher, R. Techno-economic analysis of waste-heat conversion. Joule 5, 3080-3096, doi:10.1016/j.joule.2021.10.014 (2021).

4 Pei, J., Cai, B. W., Zhuang, H. L. \& Li, J. F. Bi $2 \mathrm{Te}_{3}$-based applied thermoelectric materials: research advances and new challenges. National Science Review 7 (2020).

5 Yao, Z. et al. Solute manipulation enabled band and defect engineering for thermoelectric 
enhancements of SnTe. InfoMat 1, 571-581, doi:10.1002/inf2.12044 (2019).

6 Yang, G. S. et al. Ultra-High Thermoelectric Performance in Bulk BiSbTe/Amorphous Boron Composites with Nano-Defect Architectures. Advanced Energy Materials 10, doi: 10.1002/aenm.202000757 (2020).

7 Zhu, B. et al. Realizing record high performance in n-type $\mathrm{Bi}_{2} \mathrm{Te}_{3}$-based thermoelectric materials. Energy \& Environmental Science 13, 2106-2114, doi:10.1039/d0ee01349h (2020).

8 Wu, Z. H., Zhang, S., Liu, Z. K., Mu, E. R. \& Hu, Z. Y. Thermoelectric converter: Strategies from materials to device application. Nano Energy 91, doi: 10.1016/j.nanoen.2021.106692 (2022).

9 Huang, B., Li, G., Yang, X. \& Zhai, P. Capturing anharmonic and anisotropic natures in the thermotics and mechanics of $\mathrm{Bi}_{2} \mathrm{Te}_{3}$ thermoelectric material through an accurate and efficient potential. Journal of Physics D: Applied Physics 52, 425303, doi:10.1088/1361-6463/ab2f3a (2019).

10 Zhao, W. et al. Multi-localization transport behaviour in bulk thermoelectric materials. Nature Communications 6, 6197, doi:10.1038/ncomms7197 (2015).

11 Sarma, D. D. Essential Considerations for Reporting Thermoelectric Properties. ACS Energy Lett 6, 3715-3718, doi:10.1021/acsenergylett.1c02043 (2021).

12 Yang, C. et al. Transparent flexible thermoelectric material based on non-toxic earth-abundant p-type copper iodide thin film. Nature Communications 8, 16076, doi:10.1038/ncomms16076 (2017).

13 Jin, Q. et al. Flexible layer-structured $\mathrm{Bi}_{2} \mathrm{Te}_{3}$ thermoelectric on a carbon nanotube scaffold. Nature Materials 18, 62, doi:10.1038/s41563-018-0217-z (2019).

14 Zhang, T. et al. Mechanically Durable and Flexible Thermoelectric Films from PEDOT:PSS/PVA/Bi $0.5 \mathrm{Sb}_{1.5} \mathrm{Te}_{3}$ Nanocomposites. Advanced Electronic Materials 3, doi: 10.1002/aelm.201600554 (2017).

15 Rojas, J. P. et al. Paper-based origami flexible and foldable thermoelectric nanogenerator. Nano Energy 31, 296-301, doi:10.1016/j.nanoen.2016.11.012 (2017).

16 Park, S. H. et al. High-performance shape-engineerable thermoelectric painting. Nature Communications 7, doi:10.1038/ncomms13403 (2016).

17 Lee, J. A. et al. Woven-Yarn Thermoelectric Textiles. Advanced Materials 28, 5038-5044, doi:10.1002/adma.201600709 (2016).

18 Yan, W. et al. Thermally drawn advanced functional fibers: New frontier of flexible electronics. Materials Today, doi:10.1016/j.mattod.2019.11.006 (2020).

19 Loke, G., Yan, W., Khudiyev, T., Noel, G. \& Fink, Y. Recent Progress and Perspectives of Thermally Drawn Multimaterial Fiber Electronics. Advanced Materials 32, doi:10.1002/adma.201904911 (2020).

20 Tao, G., Stolyarov, A. M. \& Abouraddy, A. F. Multimaterial Fibers. International Journal of Applied Glass Science 3, 349-368, doi:10.1111/ijag.12007 (2012).

21 Sun, M. et al. Sn-Se alloy core fibers. Journal of Alloys and Compounds 725, 242-247, doi:10.1016/j.jallcom.2017.07.150 (2017).

22 Zhang, T. et al. High-performance, flexible, and ultralong crystalline thermoelectric fibers. Nano Energy 41, 35-42, doi:10.1016/j.nanoen.2017.09.019 (2017).

23 Sun, M. et al. Enhanced thermoelectric properties of polycrystalline $\mathrm{Bi}_{2} \mathrm{Te}_{3}$ core fibers with preferentially oriented nanosheets. APL Materials 6, doi:10.1063/1.5018621 (2018).

24 Zhang, J. et al. Single-Crystal SnSe Thermoelectric Fibers via Laser-Induced Directional 
Crystallization: From 1D Fibers to Multidimensional Fabrics. Advanced Materials 32, doi: 10.1002/adma.202002702 (2020).

25 Yan, W. et al. Advanced Multimaterial Electronic and Optoelectronic Fibers and Textiles. Advanced Materials 31, doi: 10.1002/adma.201802348 (2019).

26 Shi, X.-L., Chen, W.-Y., Zhang, T., Zou, J. \& Chen, Z.-G. Fiber-based thermoelectrics for solid, portable, and wearable electronics. Energy \& Environmental Science 14, 729-764, doi:10.1039/d0ee03520c (2021).

27 Sun, M. et al. Tailoring microstructure and electrical transportation through tensile stress in $\mathrm{Bi}_{2} \mathrm{Te}_{3}$ thermoelectric fibers. Journal of Materiomics 6, 467-475, doi:10.1016/j.jmat.2020.02.004 (2020).

28 Lu, L., Yi, W. \& Zhang, D. L. $3 \omega$ method for specific heat and thermal conductivity measurements. Review of Scientific Instruments 72, 2996-3003, doi:10.1063/1.1378340 (2001).

29 Li, L., Xu, S. \& Li, G. Enhancement of Thermoelectric Properties in Bi-Sb-Te Alloy Nanowires by Pulsed Electrodeposition. Energy Technology 3, 825-829, doi:10.1002/ente.201500071 (2015).

30 Chen, R., Lee, J., Lee, W. \& Li, D. Thermoelectrics of Nanowires. Chemical Reviews 119, 9260-9302, doi:10.1021/acs.chemrev.8b00627 (2019).

$31 \mathrm{He}$, Y. et al. Ultrahigh Thermoelectric Performance in Mosaic Crystals. Advanced Materials 27, 3639-3644, doi:10.1002/adma.201501030 (2015).

$32 \mathrm{Wu}$, Y. et al. Lattice Strain Advances Thermoelectrics. Joule 3, 1276-1288, doi:10.1016/j.joule.2019.02.008 (2019).

33 Snyder, G. J. et al. Weighted Mobility. Advanced Materials 32, doi: 10.1002/adma.202001537 (2020).

34 Wang, H. et al. Determination of the thermopower of microscale samples with an AC method. Measurement 131, 204-210, doi:10.1016/j.measurement.2018.08.027 (2019).

35 Xie, W., Tang, X., Yan, Y., Zhang, Q. \& Tritt, T. M. Unique nanostructures and enhanced thermoelectric performance of melt-spun BiSbTe alloys. Applied Physics Letters 94, doi:10.1063/1.3097026 (2009).

36 Yang, L. et al. High thermoelectric figure of merit of porous Si nanowires from 300 to $700 \mathrm{~K}$. Nature Communications 12, doi: 10.1038/s41467-021-24208-3 (2021).

37 Peng, J. \& Snyder, G. J. A figure of merit for flexibility. Science 366, 690-+, doi:10.1126/science.aaz5704 (2019).

$38 \mathrm{Li}$, X. et al. In situ physical examination of $\mathrm{Bi}_{2} \mathrm{~S}_{3}$ nanowires with a microscope. Journal of Alloys and Compounds 798, 628-634, doi:10.1016/j.jallcom.2019.05.319 (2019).

39 Huxtable, S. T. Heat transport in superlattices and nanowire arrays. Ph.D thesis, University of California, Berkeley (2002).

40 Cahill, D. G. Thermal conductivity measurement from 30 to $750 \mathrm{~K}$ : The 3 omega method (vol 61, pg 802, 1990). Review of Scientific Instruments 73, 3701-3701, doi:10.1063/1.1505652 (2002).

41 Lee, H. F. A modified $3 \omega$ method for thermal conductivity measurement of one-dimensional nanostructures. Master thesis, The Pennsylvania State University (2009).

42 Madelung, O. R., U.; Schulz, M. Non-tetrahedrally Bonded Elements and Binary Compounds. Vol. Subvolume 41C (1998).

43 Morath, C. J. M., H. J.; Cuomo, J. J.; Pappas, D. L.; Grill, A.; Patel, V. V.; Doyle, J. P.; Saenger, K. L. Picosecond optical studies of amorphous diamond and diamondlike carbon:Thermal conductivity and longitudinal sound velocity. Journal of Applied Physics, 2636 (1994).

44 Volklein, F., Reith, H. \& Meier, A. Measuring methods for the investigation of in-plane and 
cross-plane thermal conductivity of thin films. Phys Status Solidi A 210, 106-118, doi:10.1002/pssa.201228478 (2013).

45 Bodenschatz, N., Liemert, A., Schnurr, S., Wiedwald, U. \& Ziemann, P. Extending the 3 omega method: Thermal conductivity characterization of thin films. Review of Scientific Instruments $\mathbf{8 4}$, doi: 10.1063/1.4817582 (2013). 


\section{Supplementary Files}

This is a list of supplementary files associated with this preprint. Click to download.

- SupplementaryInfomation12.docx

- SupplementaryMovie1.mov 